\title{
Infrared Spectrum and Sites of Action of Sanguinarine by Molecular Mechanics and ab initio Methods
}

\author{
Ricardo Gobato ${ }^{1, *}$, Alireza Heidari \\ ${ }^{1}$ Laboratory of Biophysics and Molecular Modeling Genesis, State Secretariat for Education of Parana, Bela Vista do Paraiso, Brazil \\ ${ }^{2}$ Faculty of Chemistry, California South University, Irvine, USA
}

Email address:

ricardogobato@seed.pr.gov.br(R. Gobato),Alireza.Heidari@calsu.us (A. Heidari)

${ }^{*}$ Corresponding author

\section{To cite this article:}

Ricardo Gobato, Alireza Heidari. Infrared Spectrum and Sites of Action of Sanguinarine by Molecular Mechanics and $a b$ initio Methods. International Journal of Atmospheric and Oceanic Sciences. Vol. 2, No. 1, 2018, pp. 1-9. doi: 10.11648/j.ijaos.20180201.11

Received: April 9, 2018; Accepted: May 3, 2018; Published: May 21, 2018

\begin{abstract}
Alkaloids occupy an important position in chemistry and pharmacology. Among the various alkaloids, berberine and coralyne of the protoberberine group, sanguinarine of the benzophenanthridine group, and aristololactam-b - $d-g l u c o s i d e$ of the aristolochia group have potential to form molecular complexes with nucleic acid structures and have attracted recent attention for their prospective clinical and pharmacological utility. Sanguinarine is an alkaloid studied in the treatment of cancer cell proliferation. Found in several plants, is used in traditional medicine from several countries with Mexico and India in the natural treatment of wounds, conjunctivitis and as hallucinogen. Is a toxic quaternary ammonium salt from the group of benzylisoquinoline alkaloids. It is extracted from some plants, including bloodroot (Sanguinaria canadensis), Mexican prickly poppy (Argemone mexicana Linn) Chelidonium majus and Macleaya cordata. It is also found in the root, stem and leaves of the opium poppy but not in the capsule. Sanguinarine is a toxin that kills animal cells through its action on the $\mathrm{Na}+-\mathrm{K}+-$ ATPase transmembrane protein. Due to the diverse properties of this alkaloid, via computational methods was made using quantum chemistry to try to clarify some molecular properties that characterize its main sites of action as a drug. A study was made on a molecular structure of the sanguinarine, by Molecular Mechanics, PM3, Hartree-Fock, Density Functional Theory and Møller-Plesset. For calculations a cluster of six computers was used with Prescott-256 Celeron@ D processors. The first principles calculations have been performed to study the equilibrium configuration of Sanguinarine molecule. Several physical properties have been calculated, including formation enthalpies, entropies, dipole moments, and the infrared emission/absorption spectrum. The results showed that the main site of molecular interaction was determined to be the hydrogen atoms. This has a strong antioxidant potential in its structure. It probably interacts with free radicals reducing their carcinogenic effect on cells. A study of the infrared spectrum complemented the paper. Absorption peaks in the infrared spectrum at $1000 \mathrm{~cm}^{-1}$, for calculation MP2/6-31G and, 1240 and $1450 \mathrm{~cm}^{-1}$ for B3LYP/6-311G ** were obtained. The MP2 and B3LYP methods showed good results for the infrared absorption spectrum. Although the base used in the MP2 method is less accurate, compared to the B3LYP whose base $\mathrm{xxx}$ has more accurate and broader functionalities, they are approximately equal for frequency peaks located in the $1060.6 \mathrm{~cm}^{-1}$ and $991.1 \mathrm{~cm}^{-1}$ range.
\end{abstract}

Keywords: Alkaloids, Density Functional Theory (DFT), Hartree-Fock (HF), Molecular Geometry, Møller-Plesset (MP), Quantum Chemistry, PM3, Sanguinarine

\section{Introduction}

Sanguinarine has been shown to inhibit proliferation of several types of human cancer cell including multidrugresistant cells, whereas it has minimal cytotoxicity against normal cells such as neutrophils and keratinocytes. Is an alkaloid studied in the treatment of cancer cell proliferation
[1] Found in several plants with Argemone mexinana Linn, the plant is used in traditional medicine from several countries with Mexico and India in the natural treatment of wounds, conjunctivitis and as hallucinogen [2].

Sanguinarine (13-methyl-[1, 3]-benzodioxolo[5, 6-c]-1, 3dioxolo-[4, 5-i]-phenanthridinium chloride), Figure (1), a benzophenan-thridine alkaloid derived from the plant 
Sanguinaria canadensis, found on Argemone mexinana Linn [2] has been shown to have antimicrobial, anti-inflammatory, antioxidant, and anticancer activities [3-13].

It was reported to inhibit proliferation of different types of cancer cell, including human prostate carcinoma cells (LNCaP, PC-3 and DU145), multidrug-resistant uterine cervical carcinoma cells, human epidermoid carcinoma A431 cells, human erythroleukemia K562 cells, and the premalignant cell-line $\mathrm{HaCaT}[8,9]$. However, sanguinarine was found to be less toxic towards normal cells such as normal human epidermal keratinocytes [5].

Alkaloids occupy an important position in chemistry and pharmacology. Among the various alkaloids, berberine and coralyne of the protoberberine group, sanguinarine of the benzophenanthridine group, and aristololactam-b -dglucoside of the aristolochia group have potential to form molecular complexes with nucleic acid structures and have attracted recent attention for their prospective clinical and pharmacological utility. [14]

Dihydrosanguinarine (DHSA), a benzophenanthridines sanguinarine (SA) biosynthetic precursor and a less toxic benzophenanthridine, was also identified, based on chromatographic properties and further confirmed by gas chromatography coupled to mass spectrometry. The benzophenanthridines sanguinarine (SA) and dihydrosanguinarine (DHSA) display antimicrobiae and cytotoxic activities. These alkaloids are accumulated in roots and mature seeds, whereas berberine, a protoberberine alkaloid with antiviral properties, is accrued both in aerial and underground tissues. [15]

The alkaloids Allocryptopine, Dihydrosanguinarine, Protopine and Sanguinarine have density similar negative and positive charges. Already the main local density of positive charges are the hydrogens atoms distributed by molecular contours, and the negative oxygens atoms in its longitudinal ends, and cross for Allocryptopine and Protopine. [2]

Due to the diverse properties of this alkaloid, via computational methods was made using quantum chemistry to try to clarify some molecular properties that characterize its main sites of action as a drug. A study was made on a molecular structure of the sanguinarine, by Molecular Mechanics [16-29], PM3 [28], Hartree-Fock [28, 30, 31], Density Functional Theory [28] and Møller-Plesset [28]. For calculations a cluster of six computers was used with Prescott256 Celeron $\left(\mathrm{D}\right.$ processors ${ }^{1 .}$ The first principles calculations have been performed to study the equilibrium configuration of Sanguinarine molecule. Several physical properties have been calculated, including formation enthalpies, entropies, dipole moments, and the infrared emission/ absorption spectrum. A study of the infrared spectrum complemented the work.

The main software used for the ab initio calculations applying the quantum chemistry was the GAMMES [16-29]. Others computational dynamics software, publishing,

\footnotetext{
${ }^{1}$ CC BY-NC-SA 3.0. Creative commons. Wikipedia, The Free Encyclopedia, May 2016. [56]
}

viewing molecules were used, such as Avogadro, ChemDraw, GaussView, HyperChem, Mercury, Molden. [28]

Section 2 briefly describes the methods used in molecular and ab initio mechanics, as well as the equipment used computers. The best computational methods available are briefly explained. Obviously, the greater the function used in the calculation, the greater the requirement of the equipment to be employed, as well as the computational computation time.

In section 3 it is presented how the initial optimization geometry of the sanguinarine molecule and some of its physicochemical properties found in the liperature were performed.

It then follows to the discussions section, conclusions, tables, figures and annex. It finally presents a file in. pdb format (protein date bank), for the verification of the readers.

\section{Methods}

\subsection{Molecular Dynamics}

The great computational speed of molecular mechanics allows for its use in procedures such as molecular dynamics, conformational energy searching, and docking. All the procedures require large numbers of energy evaluations. Molecular mechanics methods are based on the following principles: Nuclei and electrons are lumped into atom-like particles; Atom-like particles are spherical (radii obtained from measurements or theory) and have a net charge (obtained from theory); Interactions are based on springs and classical potentials; Interactions must be preassigned to specific sets of atoms; Interactions determine the spatial distribution of atom-like particles and their energies;

Note how these principles differ from those of quantum mechanics. [30-34]

In short the goal of molecular mechanics is to predict the detailed structure and physical properties of molecules. Examples of physical properties that can be calculated include enthalpies of formation, entropies, dipole moments, and strain energies. Molecular mechanics calculates the energy of a molecule and then adjusts the energy through changes in bond lengths and angles to obtain the minimum energy structure. [30, 32, 33, 34]

$$
\mathrm{E}_{\mathrm{se}}=\mathrm{E}_{\text {str }}+\mathrm{E}_{\text {bend }}+\mathrm{E}_{\text {str-bend }}+\mathrm{E}_{\mathrm{oop}}+\mathrm{E}_{\text {tor }}+\mathrm{E}_{\mathrm{VdW}}+\mathrm{E}_{\mathrm{qq}}
$$

The steric energy, bond stretching, bending, stretch-bend, out of plane, and torsion interactions are called bonded interactions because the atoms involved must be directly bonded or bonded to a common atom. The Van der Waals and electrostatic (qq) interactions are between non-bonded atoms. [28, 32-37]

\subsection{Hartree Fock}

The Hartree-Fock self-consistent method is based on the one-electron approximation in which the motion of each electron in the effective field of all the other electrons is governed by a one-particle Schrodinger" equation. The 
Hartree-Fock approximation takes into account of the correlation arising due to the electrons of the same spin, however, the motion of the electrons of the opposite spin remains uncorrelated in this approximation. The methods beyond self-consistent field methods, which treat the phenomenon associated with the many-electron system properly, are known as the electron correlation methods. One of the approaches to electron correlation is the Møller-Plesset (MP) perturbation theory in which the Hartree-Fock energy is improved by obtaining a perturbation expansion for the correlation energy. [38] However, MP calculations are not variational and can produce an energy value below the true energy. [39, 40]

Another first principles approach to calculate the electronic structure for many-electron systems is the Density Functional Theory (DFT). [40] In this theory, the exchangecorrelation energy is expressed, at least formally, as a functional of the resulting electron density distribution, and the electronic states are solved for self-consistently as in the Hartree-Fock approximation. [39-42] The Density Functional Theory is, in principle, exact but, in practice, both exchange and dynamic correlation effects are treated approximately. [43]

A hybrid exchange-correlation functional is usually constructed as a linear combination of the Hartree-Fock exact exchange functional,

$$
E_{X}^{H F}=-\frac{1}{2} \sum_{i, j} \iint \Psi_{i}^{*}\left(\boldsymbol{r}_{1}\right) \Psi_{j}^{*}\left(\boldsymbol{r}_{1}\right) \frac{1}{r_{12}} \psi_{i}\left(\boldsymbol{r}_{2}\right) \psi_{j}\left(\boldsymbol{r}_{2}\right) d \boldsymbol{r}_{1} d \boldsymbol{r}_{2}
$$

and any number of exchange and correlation explicit density functionals. The parameters determining the weight of each individual functional are typically specified by fitting the functional's predictions to experimental or accurately calculated thermochemical data, although in the case of the "adiabatic connection functionals" the weights can be set a priori. [28, 44]

\subsection{B3LYP}

The B3LYP (Becke, three-parameter, Lee-Yang-Parr) [45, 46] exchange-correlation functional is:

$$
\begin{gathered}
E_{X C}^{B 3 L Y P}=E_{X}^{L D A}+a_{0}\left(E_{X}^{H F}-E_{X}^{L D A}\right)+a_{x}\left(E_{X}^{G G A}-E_{X}^{L D A}\right)+ \\
E_{C}^{L D A}+a_{c}\left(E_{c}^{G G A}-E_{c}^{L D A}\right)
\end{gathered}
$$

Are generalized gradient approximations: the Becke 88 exchange functional [47] and the correlation functional of Lee, Yang and Parr [48] for B3LYP, and $\mathrm{E}_{\mathrm{c}}^{\mathrm{DA}}$ is the VWN localdensity approximation to the correlation functional. [49]

The three parameters defining B3LYP have been taken without modification from Becke's original fitting of the analogous B3PW91 functional to a set of atomization energies, ionization potentials, proton affinities, and total atomic energies. [50]

The first principles methods $-a b$ initio (i.e. HF and DFT) discussed above can be implemented with the aid of the GAMESS set of programs to study the electronic structure and to determine the various physical properties of many- electron systems. [51] A basis set is the mathematical description of the orbitals within a system (which in turn combine to approximate the total electronic wavefunction) used to perform the theoretical calculation. [52] 3-21G, 3$21 \mathrm{G}^{*}, 6-31 \mathrm{G}, 6-31 \mathrm{G}^{*}, 6-31 \mathrm{G}^{* *}, 6-311 \mathrm{G}, 6-311 \mathrm{G}^{*}$, 6$311 \mathrm{G}^{* *}$ are the basis sets used in the calculations. The functional Becke-style one parameter functional using modified Perdew-Wang exchange and Perdew-Wang 91 correlation is used for DFT Calculations. [43, 53]

The SCF method and extensions to it are mathematically and physically considerably more complicated than the oneelectron methods already discussed. Thus, one normally does not perform such calculations with pencil and paper, but rather with complicated computer programs. Terms like "Hartree-Fock" or "correlation energy" have specific meanings and are pervasive in the literature. [54]

The vast literature associated with these methods suggests that the following is a plausible hierarchy:

$$
\mathrm{HF}<<\mathrm{MP} 2<\mathrm{CISD}<\mathrm{CCSD}<\mathrm{CCSD}(\mathrm{T})<\text { FCI }
$$

The extremes of 'best', FCI, and 'worst', HF, are irrefutable, but the intermediate methods are less clear and depend on the type of chemical problem being addressed. [55]

For calculations a cluster of 6 computer models was used: Prescott-256 Celeron(C) D processors, [56] featuring double the L1 cache (16 KB) and L2 cache $(256 \mathrm{~KB})$, Socket 478 clock speeds of $2.13 \mathrm{GHz}$; Memory DDR2 PC4200 512MB; Hitachi HDS728080PLAT20 80 GB and CD-R.

The dynamic was held in Molecular Mechanics Force Field $(\mathrm{Mm}+)$, Equation (1), after the quantum computation was optimized via PM3 [57-61] and then by DFT, [30, 43] with functional B3LYP [62] and base 6-311G** [30, 43, 51]. The molecular dynamics at algorithm Polak-Ribiere [63], conjugate gradient, at the termination condition: RMS gradient [64] of $0.1 \mathrm{kcal} / \mathrm{A}$. mol or 405 maximum cycles in vacuum.

The first principles calculations have been performed to study the equilibrium configuration of Sanguinarine molecule using the Hyperchem 7.5 Evaluation [65], Gaussview v.5 a general molecular and electronic structure processing program, an advanced semantic chemical editor, visualization, and analysis platform [66] and GAMESS is a computational chemistry software program and stands for General Atomic and Molecular Electronic Structure System [51] set of programs. The first principles approaches can be classified into two main categories: the Hartree-Fock approach and the density functional approach. [39]

\section{Fundamentals}

\subsection{Geometry Optimization}

The dynamic was held in Molecular Mechanics Force Field $(\mathrm{Mm}+)$, Equation (1), computed geometry optimization molecular at algorithm Polak-Ribiere [63], conjugate gradient, at the termination condition: RMS gradient [64] of 
$0.1 \mathrm{kcal} / \mathrm{A}$. mol or 405 maximum cycles in vacuum. Molecular properties: electrostatic potential 3D mapped isosurface, mapped function range, minimum 0.144 at maximum 0.734 and minimum -0.008 at maximum +0.216 , $\mathrm{Mm}+$ and PM3 methods, respectively. For display range legend, from positive color lime green to negative color pink, total charge density contour value of 0.05 , gourand shaded surface.

\subsection{Chemical Formula and Physicochemical Property of Sanguinarine}

Sanguinarine is a toxic quaternary ammonium salt from the group of benzylisoquinoline alkaloids. It is extracted from some plants, including bloodroot (Sanguinaria canadensis), Mexican prickly poppy Argemone mexicana [67], Chelidonium majus and Macleaya cordata. It is also found in the root, stem and leaves of the opium poppy but not in the capsule. Sanguinarine is a toxin that kills animal cells through its action on the $\mathrm{Na}+\mathrm{K}+$-ATPase transmembrane protein [68]. Epidemic dropsy is a disease that results from ingesting sanguinarine [69]. If applied to the skin, sanguinarine kills cells and may destroy tissue. In turn, the bleeding wound may produce a massive scab, called an eschar. For this reason, sanguinarine is termed an escharotic. [70]

The molecule has: CAS No. 2447-54-3; Chemical Name: Sanguinarine; Synonyms:13-Methyl-[1, 3]benzodioxolo[5, 6c]-1, 3-dioxolo[4, 5-i]phenanthridinium; Molecular Formula: $\mathrm{C}_{2} \mathrm{OH}_{14} \mathrm{NO}_{4}$; Molar mass: 332.3295; Density: $0.0184 \mathrm{~g} / \mathrm{mol}$; Melting point: $205-215^{\circ} \mathrm{C}$; Ecotoxicology: $\mathrm{LD}^{50} ; 19.400$
$\mathrm{mgDkg}^{-1}$ (mouse, intravenous) [71]; $80 \mathrm{mgDkg}^{-1}$ (mouse, subcutaneous) [72]; $18 \mathrm{mgDkg}^{-1}$ (mouse, Intraperitoneal); [73] Solubility: soluble in alcohol, chloroform, acetone, ethyl acetate; UVmax: 234, 283, $325 \mathrm{~nm}$ in methyl alcohol. [37, $74,75]$.

\section{Discussions}

The Figures (1-b) and (1-d) show the distribution of charges in the sanguinarine molecule. The Figure (1-b) represents the molecular dynamics by the $\mathrm{Mm}+$ method, according to Equation (1). The charges range from 0.144, in pink, to 0.734 , in lime green, to the distribution of charges in the molecule.

The Figure (1-d) represents the molecular dynamics by the PM3 method. The load distribution in the molecule ranges from -0.008 negative, in pink, to +0.216 positive, in lime green, respectively.

By the $\mathrm{Mm}+$ method, Figure (1-b), this indicates that the molecule has a positive potential, having a positive variation of charge distribution, $\Delta \delta=+0.59$, being strongly antioxidant. Likewise in Figure (1-d), by the PM3 method, a positive charge distribution variation, $\Delta \delta=+0.224$, of lesser intensity, but more suitably distributed, occurs. This method represents the most appropriate displacement of charges in the molecule. As a result we have a better view of the action sites of the molecule. The $\mathrm{Mm}+$ method is inappropriate for the representation of the displacements of charges in the molecule, but efficient in the deduction of an antioxidant molecule.

Table 1. Thermochemical parameters of the molecule Sanguinarine obtained by ab initio methods [28].

\begin{tabular}{|c|c|c|c|}
\hline \multirow{3}{*}{ Methods/Base } & \multicolumn{3}{|c|}{ Thermochemistry } \\
\hline & ${ }^{\mathbf{E}}$ T hermal & CV & $\mathbf{S}$ \\
\hline & (Kcal/mol) & (cal/mol. K) & (cal/mol. K) \\
\hline B3LYP/6-311G** & 198.160 & 76.253 & 144.017 \\
\hline $\mathrm{HF} / 6-21 \mathrm{G}$ & 212.458 & 70.198 & 137.874 \\
\hline MP2/6-31G & 200.357 & 76.830 & 144.219 \\
\hline
\end{tabular}

In Figure (1-d) it can be verified that the sites of antioxidant action are localized and distributed in the hydrogen atoms throughout the length of the molecule, presenting a strong electric potential of interaction in these sites. The nitrogen atom at the center of the molecule exerts the potential for moderate interaction compared to the hydrogens. Already the four oxygen atoms, located at both ends of the molecule, distributed two by two, have a negative potential, -0.008, represented in pink, also providing an antioxidant interaction, free radicals.

Although the $\mathrm{Mm}+$ and PM3 methods [16-28] are less sophisticated with others, with more accurate calculations, they give us an adequate vision for what the study proposed, and to determine the main sites of action of sanguinarine.

Analyzing the infrared spectrum, Figure (2), sanguinarine has absorption peaks at the frequencies $3009.4,2984.3 \mathrm{~cm}^{-1}$ and 1501.1, 1471.0, 1275.5 and $1060.6 \mathrm{~cm}^{-1}$ for the method/base MP2/6-31G [28].

Analyzing the infrared spectrum, Figure (3), sanguinarine has absorption peaks at the frequencies $3115.3,3109.7 \mathrm{~cm}^{-1}$ and 1484.6, $1295.4 \mathrm{~cm}^{-1}$ and $1027.5,991.1 \mathrm{~cm}^{-1}$ for the $\mathrm{method} /$ base, B3LYP/6-311G** [28, 31, 34, 42, 51]. 
a)
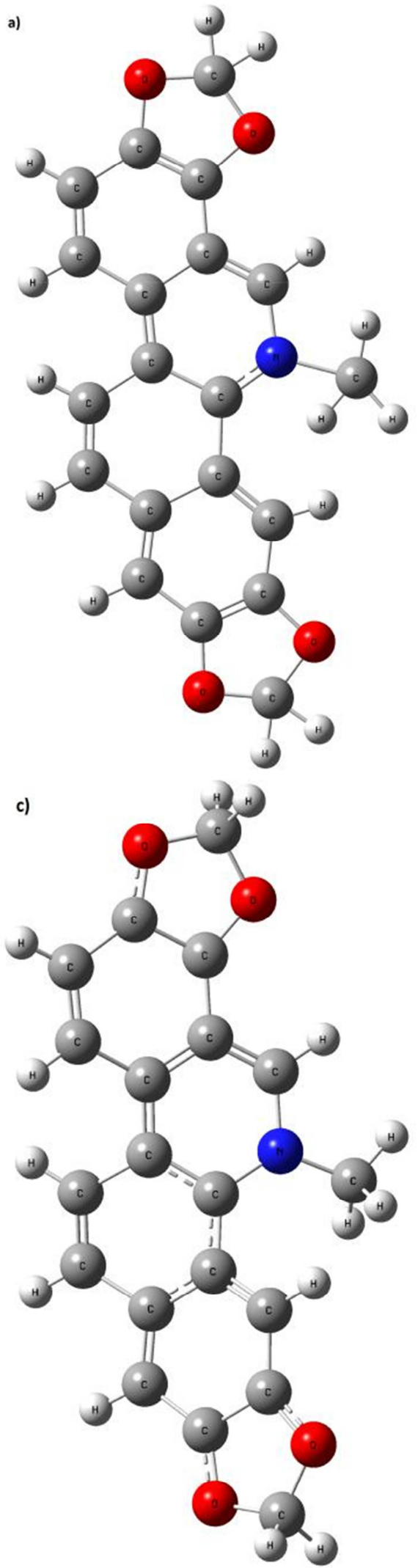

b)

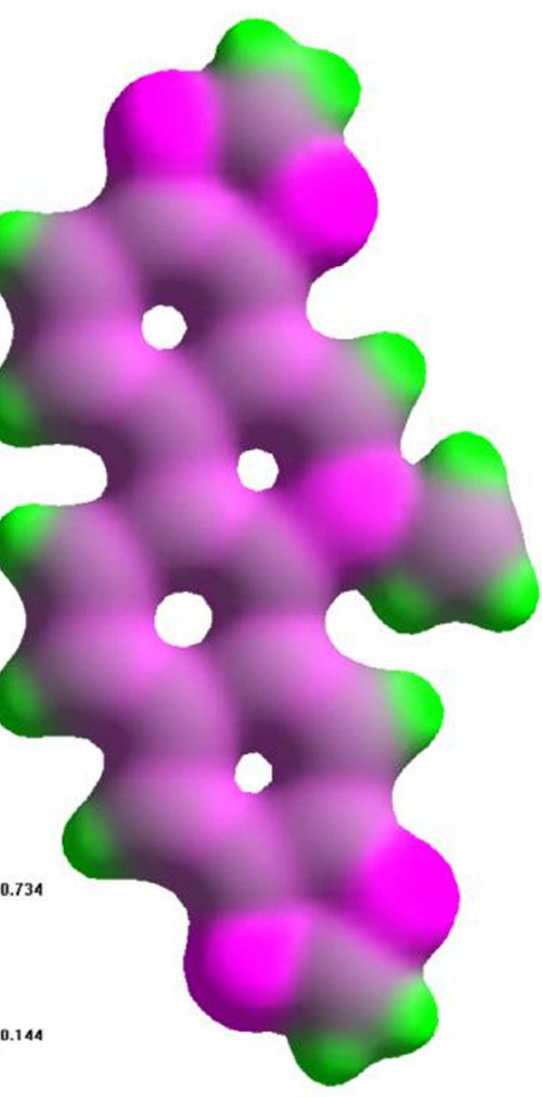

d)

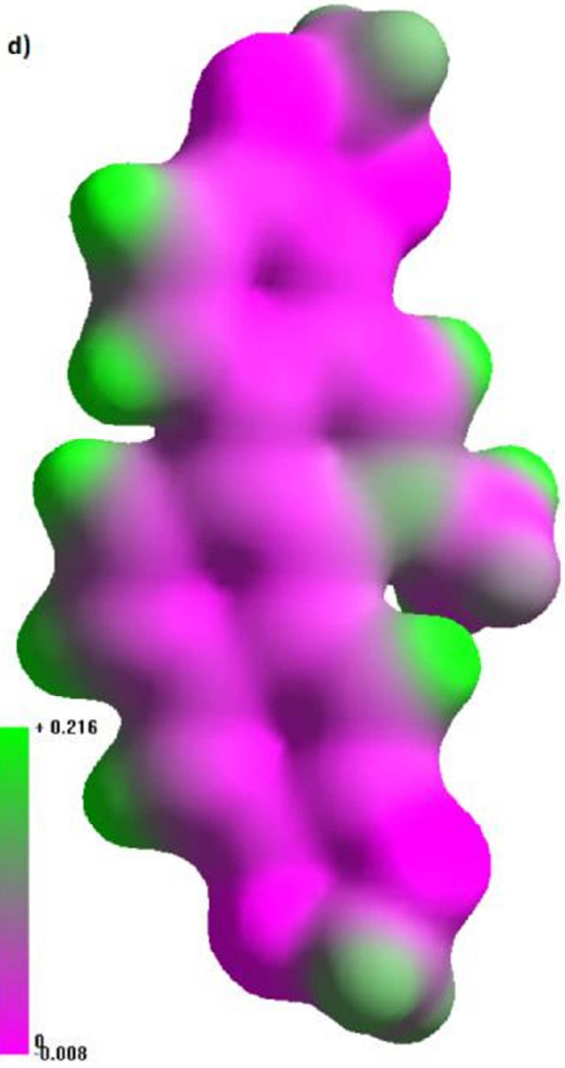

Figure 1. Above the Figures (a) and (b) representation of the molecular structure of Sanguinarine, obtained through computer via Molecular Mechanics Mm+ calculation and optimized [30, 31, 32, 33, 34] obtained using computer programs HyperChem 7.5 Evaluation [65]. Below the Figures (c) and (d) representation of the molecular structure of Sanguinarine, obtained through computer via and then its geometry was optimized via PM3 [57, 58, 59, 60, 61] obtained using computer programs HyperChem 7.5 obtained using computer programs GAMESS [51]. Images obtained in the softwares HyperChem 7.5 Evaluation [65] and Avogadro [76]. 
Ricardo Gobato and Alireza Heidari: Infrared Spectrum and Sites of Action of Sanguinarine by Molecular Mechanics and ab initio Methods

Table 2. Table containing the dipole moments of the Sanguinarine molecule via ab initio methods [28].

\begin{tabular}{|c|c|c|c|c|}
\hline \multirow{2}{*}{ Methods/Base } & \multicolumn{4}{|c|}{ Dipole moment (Debye) } \\
\hline & $\mathbf{X}$ & $\mathbf{Y}$ & $\mathbf{Z}$ & Total \\
\hline $\mathrm{UHF} / 6-31 \mathrm{G}$ & 0.5075 & -0.1448 & 0.9548 & 1.0910 \\
\hline UBLYP/STO-3G & 1.7949 & -2.0135 & 0.7058 & 2.7882 \\
\hline UB3LYP/6-311** & 1.9087 & -1.5920 & -0.0810 & 2.4868 \\
\hline $\mathrm{UHF} / 3-21 \mathrm{G}$ & 0.5075 & -0.1448 & 0.9549 & 1.0910 \\
\hline $\mathrm{UHF} / 6-311 \mathrm{G}^{* *}$ & -1.2776 & -1.6030 & -0.2325 & 2.0630 \\
\hline UMP2-FC/STO-3G & -1.8508 & -1.4547 & -0.1789 & 2.3609 \\
\hline UMP2-FC/6-31G & 0.6840 & -0.6053 & 0.8109 & 1.2214 \\
\hline B3LYP/STO-3G & 1.7949 & -2.0135 & 0.7058 & 2.7882 \\
\hline
\end{tabular}

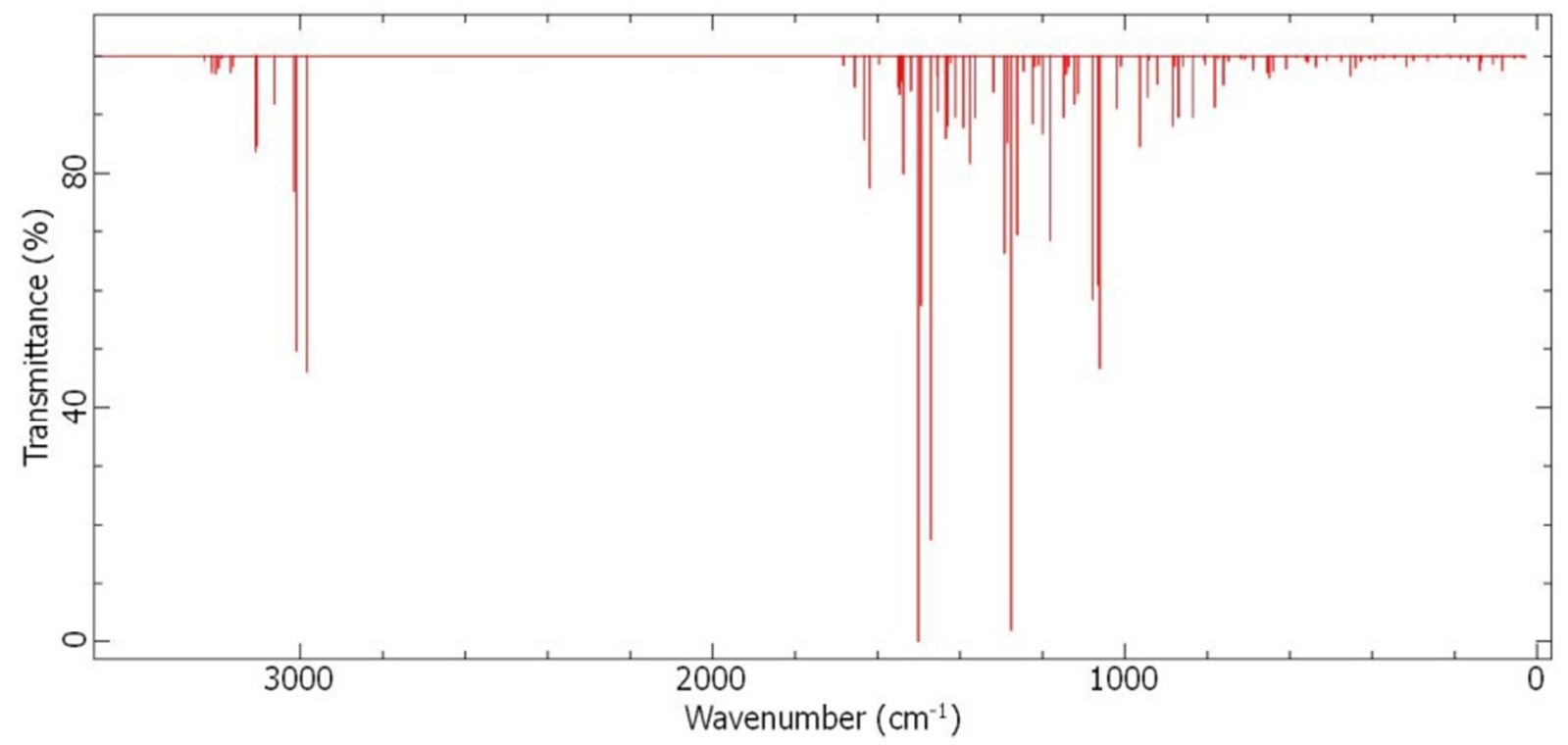

Figure 2. The above figure represents the transmittance (\%) in function with wavelength $\mathrm{cm}^{-1}$ for the infrared spectrum of the sanguinarine molecule, after optimization of the geometry with the method/base, B3LYP/6-311G** [28, 31, 34, 42, 51, 63] obtained using computer programs GAMESS [51]. The image was generated using the Avogadro program. [76].

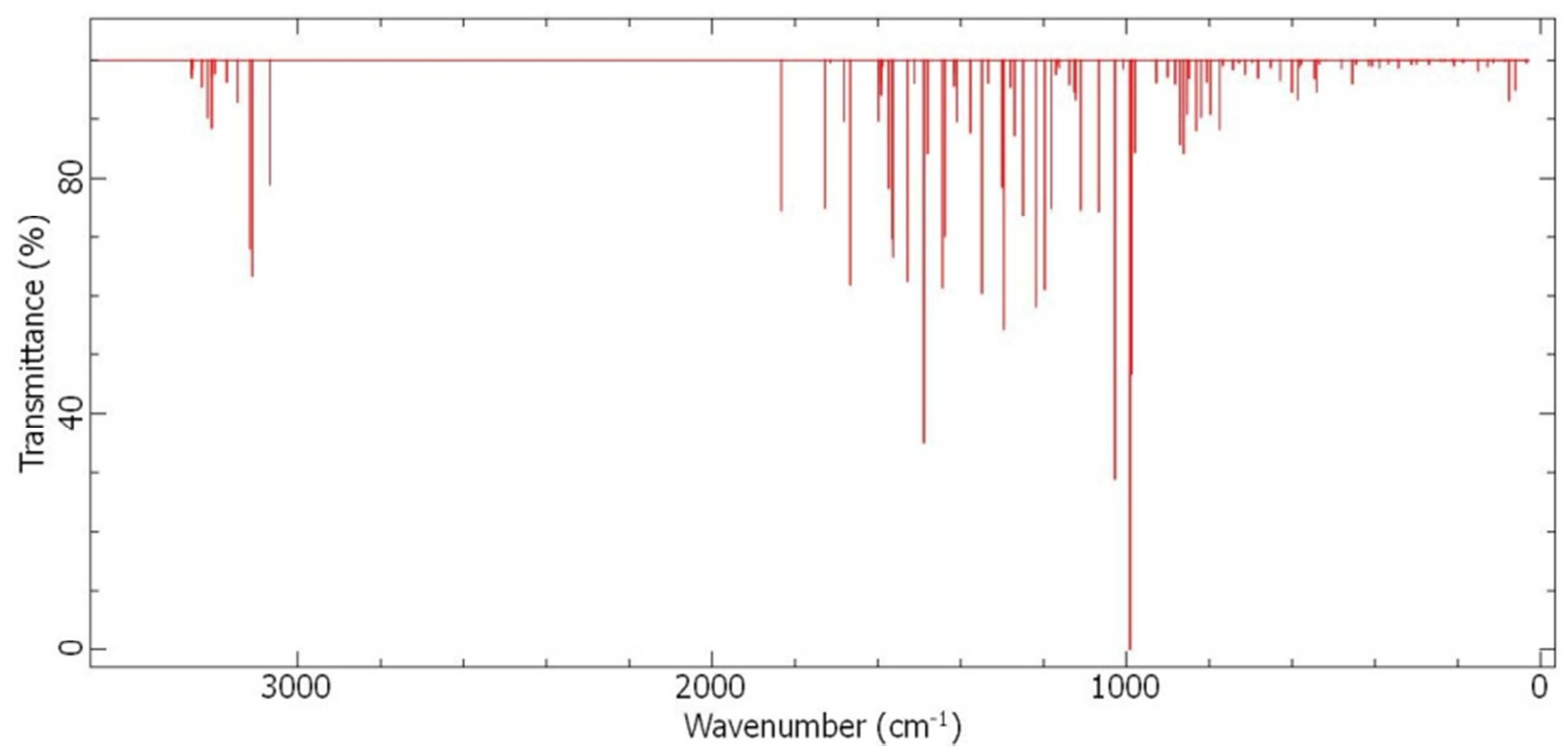

Figure 3. The above figure represents the transmittance (\%) in function with wavelength $\mathrm{cm}^{-1}$ for the infrared spectrum of the sanguinarine molecule, after optimization of the geometry with the method/base, MP2/6-31G [28, 76, 77] obtained using computer programs GAMESS [51]. The image was generated using the Avogadro program. [76]. 


\section{Conclusions}

The main sites of interactions of the molecule was finded. This has a strong antioxidant potential in its structure. It probably interacts with free radicals reducing their carcinogenic effect on cells.

The sites of antioxidant action are localized and distributed in the hydrogen atoms throughout the length of the molecule, presenting a strong electric potential of interaction in these sites. The nitrogen atom at the center of the molecule exerts the potential for moderate interaction compared to the hydrogens. Already the four oxygen atoms, located at both ends of the molecule, distributed two by two, also providing an antioxidant interaction, free radicals.

The infrared spectrum for the method/base, MP2/6-31G has absorption peaks at the frequencies main $1060.6 \mathrm{~cm}^{-1}$ and for the method/base, B3LYP/6-311G** 1027.5 and $991.1 \mathrm{~cm}^{-}$ 1 , being approximately equal in both methods.

The MP2 and B3LYP methods showed good results for the infrared absorption spectrum. Although the base used in the MP2 method is less accurate, compared to the B3LYP whose base $6-311 \mathrm{G}^{* *}$ has more accurate and broader functionalities, they are approximately equal for frequency peaks located in the $1060.6 \mathrm{~cm}^{-1}$ and $991.1 \mathrm{~cm}^{-1}$ range.

\section{References}

[1] M. Lopus and D. 1 Panda. The benzophenanthridine alkaloid sanguinarine perturbs microtubule assembly dynamics through tubulin binding. A possible mechanism for its antiproliferative activity. The FEBS Journal Federation of European Biochemical Societies, 273(10):2139-2150, May 2006.

[2] R. Gobato; D. F. G. Fedrigo and A. Gobato. Molecular geometry of alkaloids present in seeds of mexican prickly poppy. Cornell University Library. Quantitative Biology, Jul 15, 2015. arXiv:1507. 05042.

[3] K. C. Godowski. Antimicrobial action of sanguinarine. J. Clin. Dent., (1):96-101, 1989.

[4] T. K. Beuria; M. K. Santra and D. Panda. Sanguinarine blocks cytokinesis in bacteria by inhibiting FtsZ assembly and bundling. Biochemistry, (44):16584-16593, 2005.

[5] N. Ahmad; S. Gupta; M. M. Husain; K. M. Heiskanen and H. Mukhtar. Differential anti-proliferative and apoptotic response of sanguinarine for cancer cells versus normal cells. Clin. Cancer Res., (6):6, 1424-1428, 2000.

[6] J. Slaninova; E. Taborska; Bochorakoa and J. Slanina. Interaction of benzophenanthridine and protoberberine alkaloids with animal and yeast cells. Cell Biol. Toxicol., (17):51-63, 2001.

[7] P. Weerasinghe; S. Hallock and A. Liepins. Bax, [b]cl-2, and [nf]-kappa[b] expression in sanguinarine induced bimodal cell death. Exp. Mol. Pathol., (71):89-98, 2001.

[8] Z. Ding; S. C. Tang; P. Weerasinghe; X. Yang, A. Pater and A. Liepins. The alkaloid sanguinarine is effective against multidrug resistance in human cervical cells via bimodal cell death. Biochem. Pharmacol, (63):1415-1421, 2002.
[9] V. M. Adhami; M. H. Aziz; H. Mukhtar and N. Ahmad. Activation of prodeath Bcl-2 family proteins and mitochondrial apoptosis pathway by sanguinarine in immortalized human HaCaT keratinocytes. Clin. Cancer Res., (9):3176-3182, 2003.

[10] V. M. Adhami; M. H. Aziz; S. R. Reagan-[S]haw; M. Nihal; H. Mukhtar and N. Ahmad. Sanguinarine causes cell cycle blockade and apoptosis of human prostate carcinoma cells via modulation of cyclin kinase inhibitor-cyclin-cyclin-dependent kinase machinery. Mol. Cancer Ther., (3):933-940, 2004.

[11] A. Vogt; A. Tamewitz, J. Skoko; R. P. Sikorski; K. A. Giuliano and J. S. Lazo. The benzocphenanthridine alkaloid, sanguinarine, is a selective, cell-active inhibitor of mitogenactivated protein kinase phosphatase-1. J. Biol. Chem., (280):19078-19086, 2005.

[12] J. P. Eun and G. Y. Koh. Suppression of angiogenesis by the plant alkaloid, sanguinarine. Biochem. Biophys. Res. Commun., (317):618-624, 2004.

[13] M. P \& U. J. Dvorak et al.. Differential effects of selected natural compounds with anti-inflammatory activity on the glucocorticoid receptor and $[\mathrm{nf}]-\mathrm{kappa}[\mathrm{b}]$ in $[\mathrm{h}] \mathrm{e}[\mathrm{l}] \mathrm{a}$ cells. Chem. Biol. Interact., (159):117-128, 2006.

[14] M. Maiti and G. S. Kumar. Molecular aspects on the interaction of protoberberine, benzophenanthridine, and aristolochia group of alkaloids with nucleic acid structures and biological perspectives. Medicinal Research Reviews, 27(5):649-695, September 2007.

[15] M. Monforte-Gonzalez; C. Guízar-Gonzalez; K. TrujilloVillanueva and F. Vazquez-Flota. Sanguinarine and Dihydrosanguinarine Accumulation in Argemone mexicana (1) Cell Suspension Cultures Exposed to Yeast Extract. J. Mex. Chem. Soc., 56(1):19-22, 2012. Sociedad Qu'imica de Mexico'. ISSN 1870-249X.

[16] R. Gobato; A. Gobato and D. F. G. Fedrigo. Inorganic arrangement crystal beryllium, lithium, selenium and silicon. In XIX Semana da Física. Simposio Comemorativo dos 40 anos do Curso de Física da Universidade Estadual de Londrina, Universidade Estadual de Londrina (UEL): Londrina, Brasil, 2014.

[17] R. Gobato; D. F. G. Fedrigo and A. Gobato. Allocryptopine, Berberine, Chelerythrine, Copsitine, Dihydrosanguinarine, Protopine and Sanguinarine. Molecular geometry of the main alkaloids found in the seeds of Argemone mexicana Linn. Parana J. Sci. Edu., 1(2):7-16, December 2015.

[18] R. Gobato; D. F. G. Fedrigo and A. Gobato. Avro: key component of Lockheed X-35. Parana J. Sci. Educ., 1(2):1-6, December 2015.

[19] R. Gobato; A. Gobato and D. F. G. Fedrigo. Inorganic arrangement crystal beryllium, lithium, selenium and silicon. Cornell University Library. arXiv. org, Atomic and Molecular Clusters (physics. atm-clus); Materials Science (cond-mat. mtrl-sci). Aug 01, 2015.

[20] R. Gobato; D. F. G. Fedrigo and A. Gobato. LOT-G3: Plasma Lamp, Ozonator and Cw Transmitter. Ciencia e Natura, 38(1), 2016.

[21] R. Gobato. Matter and energy in a non-relativistic approach amongst the mustard seed and the faith. A metaphysical conclusion. Parana J. Sci. Educ., 2(3):1-14, March 2016. 
[22] R. Gobato; A. Gobato and D. F. G. Fedrigo. Harnessing the energy of ocean surface waves by Pelamis System. Parana $J$. Sci. Educ., 2(2):1-15, February 2016.

[23] R. Gobato; A. Gobato and D. F. G. Fedrigo. Study of the molecular electrostatic potential of D-Pinitol an active hypoglycemic principle found in Spring flower Three Marys (Bougainvillea species) in the $\mathrm{Mm}+$ method. Parana J. Sci. Educ., 2(4):1-9, May 2016.

[24] R. Gobato; A. Gobato and D. F. G. Fedrigo. Mathematics for input space probes in the atmosphere of Gliese 581d. Parana J. Sci. Educ., 2(5):6-13, July 2016.

[25] A. Gobato R. Gobato and D. F. G. Fedrigo. Study of tornadoes that have reached the state of Parana. Parana J. Sci. Educ., 2(1):1-27, 20162016.

[26] R. Gobato. Study of the molecular geometry of Caramboxin toxin found in star flower (Averrhoa carambola L.). Parana $J$. Sci. Educ., 3(1):1-9, January 2017.

[27] R. Gobato and M. Simões F. Alternative Method of RGB Channel Spectroscopy Using a CCD Reader. Ciencia $e$ Natura, 39(2), 2017.

[28] R. Gobato and A. Heidari. Calculations Using Quantum Chemistry for Inorganic Molecule Simulation BeLi2SeSi. Science Journal of Analytical Chemistry, 5(5):76-85, September 2017.

[29] M. R. R. Gobato; R. Gobato and A. Heidari. Planting of Jaboticaba Trees for Landscape Repair of Degraded Area. Landscape Architecture and Regional Planning, 3(1):1-9, March Jan. 18, 2018.

[30] I. N. Levine. Quantum Chemistry. Pearson Education (Singapore) Pte. Ltd., Indian Branch, 482 F. I. E. Patparganj, Delhi 110 092, India, 5th ed. edition, 2003.

[31] E. Eliav. Elementary introduction to Molecular Mechanics and Dynamics, Jun 2013.

[32] Thomas W. Shattuck. Colby College Molecular Mechanics Tutorial. Department of Chemistry, Colby College, Waterville, Maine 04901, September 2008.

[33] W. D. Cornell; P. Cieplak; C. I. Bayly; I. R. Gould; K. M. Merz Jr.; D. M. Ferguson; D. C. Spellmeyer; T. Fox; J. W. Caldwell and P. A. Kollman. A Second Generation Force Field for the Simulation of Proteins, Nucleic Acids, and Organic Molecules. J. Am. Chem. Soc., 117:5179-5197, 1995.

[34] W. J. Hehre. A Guide to Molecular Mechanics and Quantum Chemical Calculations, Wavefunction. Inc., Irvine, CA, 2003.

[35] R. Gobato. Benzocaína, um estudo computacional. Master's thesis, Universidade Estadual de Londrina (UEL), 2008.

[36] R. Gobato; A. Gobato and D. F. G. Fedrigo. Molecular electrostatic potential of the main monoterpenoids compounds found in oil Lemon Tahiti - (Citrus Latifolia Var Tahiti). Parana J. Sci. Educ.,, 1(1):1-10, Nov. 2015.

[37] R. Gobato; D. F. G. Fedrigo and A. Gobato. Allocryptopine, Berberine, Chelerythrine, Copsitine Dihydrosanguinarine, Protopine and Sanguinarine. Molecular geometry of the main alkaloids found in the seeds of Argemone Mexicana Linn. Parana J. Sci. Educ., 1(2):7-16, Dec. 2015.

[38] A. Szabo and N. S. Ostlund. Modern Quantum Chemistry. Dover Publications, New York, 1989.
[39] K. Ohno; K. Esfarjani and Y. Kawazoe. Computational Material Science. Springer-Verlag, Berlin, 1999.

[40] K. Wolfram and M. C. Hothausen. Introduction to DFT for Chemists. John Wiley \& Sons, Inc. New York, 2nd ed. edition, 2001.

[41] P. Hohenberg and W. Kohn. Inhomogeneous Electron Gas. Phys. Rev., (136):B864-B871, 1964.

[42] W. Kohn and L. J. Sham. Self-Consistent Equations Including Exchange and Correlation Effects. Phys. Rev., (140): A1133, 1965.

[43] J. M. Thijssen. Computational Physics. Cambridge University Press, Cambridge, 2001.

[44] J. P. Perdew; M. Ernzerhof and K. Burke. Rationale for mixing exact exchange with density functional approximations. J. Chem. Phys., 105(22):9982-9985, 1996.

[45] K. Kim and K. D. Jordan. Comparison of Density Functional and MP2 Calculations on the Water Monomer and Dimer. $J$. Phys. Chem., 40(98):10089-10094, 1994.

[46] P. J. Stephens; F. J. Devlin; C. F. Chabalowski and M. J. Frisch. Ab Initio Calculation of Vibrational Absorption and Circular Dichroism Spectra Using Density Functional Force Fields. J. Phys. Chem., 45(98):11623-11627, 1994.

[47] A. D. Becke. Density-functional exchange-energy approximation with correct asymptotic behavior. Phys. Rev. A., 38(6):3098-3100, 1988.

[48] C. Lee; W. Yang and R. G. Parr. Development of the ColleSalvetti correlation-energy formula into a functional of the electron density. Phys. Rev. B, 37(2):785-789, 1988.

[49] S. H. Vosko; L. Wilk and M. Nusair. Accurate spin-dependent electron liquid correlation energies for local spin density calculations: a critical analysis. Can. J. Phys., 58(8):12001211,1980

[50] A. D. Becke. Density-functional thermochemistry. The role of exact exchang. J. Chem. Phys., 98(7):5648-5652, 1993.

[51] M. S. Gordon et al. General Atomic and Molecular Electronic Structure System (GAMESS). J. Comput. Chem., 14:13471363, 1993.

[52] J. B. Foresman and Æleen Frisch. Exploring Chemistry with Electronic Structure Methods. Gaussian, Inc. Pittsburgh, PA, 2nd ed edition, 1996.

[53] L. Mainali; D. R. Mishra and M. M. Aryal. First Principles Calculations to Study the Equilibrium Configuration of Ozone Molecule. Department of Biophysics. Medical College of Wisconsin. 8701 Watertown Plank Road. Milwaukee, WI 53226.

[54] J. P. Lowe and K. A. Peterson. Quantum Chemistry. Elsevier Inc., 3th, San Diego, CA, USA. Theobalds Road, London WC1X 8RR, UK. 2006.

[55] J. J. W. McDouall. Computational Quantum Chemistry. Molecular Structure and Properties in Silico. The Royal Society of Chemistry, Thomas Graham House, Science Park, Milton Road, Cambridge CB4 OWF, UK, 2013.

[56] CC BY-NC-SA 3.0. Creative commons. Wikipedia, The Free Encyclopedia, May 2016. 
[57] M. J. Frisch; G. Scalmani; T. Vreven and G. Zheng. Analytic second derivatives for semiempirical models based on MNDO. Mol. Phys., 2009.

[58] W. Thiel and A. A. Voityuk. Extension of MNDO to d orbitals: Parameters and results for the second-row elements and for the zinc group. J. Phys. Chem., (100):616-26, 1996.

[59] W. Thiel and A. A. Voityuk. Extension of the MNDO formalism to $\mathrm{d}$ orbitals: Integral approximations and preliminary numerical results. Theor. Chem. Acc., (81):391404, 1992.

[60] J. J. P. Stewart. Optimization of parameters for semiempirical methods. I. Methods. J. Comp. Chem., (10):209-20, 1989.

[61] J. J. P. Stewart. Optimization of parameters for semiempirical methods. II. Applications. J. Comp. Chem., (10):221-64, 1989.

[62] W. Yang C. Lee and R. G. Parr. Phys. Rev. B, 37:785-789, 1988.

[63] E. Polak. Computational Methods in Optimization, v. 77. Elsevier, 111 Fifth Av., New York, New York 10003, 1971.

[64] Anthony K. Rappe and Carla J. Casewit. Molecular Mechanics Across Chemistry. University Science Books, 55D Gate Five Road, Sausalito, CA 94965, 1952(1997).

[65] Computational Chemistry Software. Hyperchem 7.5 Evaluation. Hypercube, Inc., 2003.

[66] R. Dennington; T. Keith and J. Millam. Gaussview, Version 5, 2009.

[67] A. C. Santos and P. Adkilen. The Alkaloids of Argemone Mexicana. Journal of the American Chemical Society, 54(7):2923-2924, 1932.
[68] B. J. R. Pitts and L. R. Meyerson. Inhibition of Na, K-ATPase Activity and Ouabain Binding by Sanguinarine. Drug Development Research, 1(1):43-49, 1981.

[69] Das M. and S. K. Khanna. Clinicoepidemiological, Toxicological, and Safety Evaluation Studies on Argemone Oil. Critical Reviews in Toxicology, 27(3):273-297, 1997.

[70] J. J. Cienki and L. Zaret. An Internet Misadventure: Bloodroot Salve Toxicity. The Journal of Alternative and Complementary Medicine, 16(10): 1125-1127, 2010.

[71] Farmakologiya i Toksikologiya, v. 29. PMID, 1966. p. 76.

[72] Arzneimittel-Forschung. Drug Research, v. 10. PMID, 1960. p. 135.

[73] J. Berdy' et al. Handbook of Antibiotic Compounds, v. I-X. Boca Raton, Florida, USA, 1980-1982. p. 195.

[74] NCBI. PubChem. PubChem Compound. NCBI. National Center for Biotechnology Information. $<$ http://pubchem.ncbi.nlm.nih.gov>.

[75] The Merck Index. p. 65. Rahway: Merck \& Co, 10th ed. edition, 1983.

[76] M. D. Hanwell; D. E. Curtis; D. C. Lonie; T. Vandermeersch; E. Zurek and G. R. Hutchison. Avogadro: an advanced semantic chemical editor, visualization, and analysis platform. J. Cheminform, 17(4), Aug. 13, 2012.

[77] W. J. Hehre; R. F. Stewart and J. A. Pople. Self-Consistent Molecular Orbital Methods. Use of Gaussian expansions of Slater-type atomic orbitals. J. Chem. Phys., (51):2657-64, 1969. 